\title{
Actuaciones tras el bombardeo de Alicante en 1691 La fortificación temporal de Bernet y Herrera
} Ángel Benigno González Avilés ${ }^{\mathrm{a}}$, María Isabel Pérez Millán ${ }^{\mathrm{b}}$, Roberto Yáñez Pacios ${ }^{\mathrm{a}}$

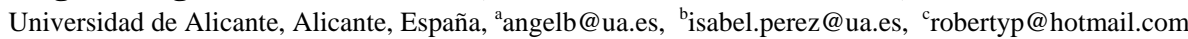

\begin{abstract}
After the start of the bastion of San Carlos as the first defensive element of Alicante's fortification, it was essential to undertake the second of the points that was urgently in the city, its provisional defense. Thanks to a letter dated November $10^{\text {th }}$ of 1691 , we know that the field master Don Simon Bernet and Sergeant Don Diego de Herrera Davila acknowledged the city and they arranged everything to execute urgently "with the short time allowed and the resources to the most necessary as a paper which has been formed."

Simon Bernet presented the proposal to the king. He expressed the town regret by not remedy its situation more than three months after the fatal bombing. Also he mentioned the fear of a new offensive by the French fleet and the inability to defend the city for more than an hour. Everything was evidenced by the recognition of the city by Sergeant Don Diego de Herrera.

Unfortunately, only the letter of Bernet and Herrera has come down to us. Thanks to it we can find out the intentions of the project to prevent the landing on the beach of Bavel.
\end{abstract}

Keywords: Alicante, ingenieros militares, Bernet y Herrera, fortificación temporal.

\section{Introducción}

El siglo XVII supuso un estancamiento general a lo largo de la historia de las fortificaciones de Alicante. La ausencia de conflictos bélicos y las crisis económicas de la Corona desencadenaron en una etapa sombría para el desarrollo de su infraestructura defensiva y el paso del tiempo arruinó las obsoletas fortificaciones. Las inversiones en materia defensiva en la plaza alicantina cayeron en picado. El frente defensivo y la importancia estratégica cambiaba de ubicación y Alicante quedó relegada a un segundo rango que la condenó el resto de su historia. Esta condición y la inexpugnabilidad de su fortaleza marcaron el devenir de todas las actuaciones defensivas del siglo XVII y XVIII. Episodios como el bombardeo del 11 de junio 1691, de manos de la armada francesa bajo las órdenes del comandante D'Estreés y la nula atención de la corona por recuperar las fortificaciones de la llave del reino -así se denominada a Alicante-, plasmó la inoperancia de sus defensas.

Ninguno de los numerosos proyectos planteados para la ciudad de Alicante en el siglo XVII, se llevó a cabo. Tan sólo el Baluarte de San Carlos, planteado en diferentes proyectos de fortificación de la plaza por diferentes ingenieros como Castellón y Valero (González, 2014) y Ambrosio Borçano (Pérez, 2014), ubicado en la desembocadura del barranco de San Blas, capaz de repeler un ataque por mar, se convertiría en el único elemento defensivo ejecutado, precisamente para evitar de nuevo un suceso tan demoledor como el que había protagonizado el bombardeo francés.

La construcción del Baluarte de San Carlos fue acompañada por la ejecución de una 
construcción defensiva provisional, que ante la eminencia de un nuevo ataque enemigo pudiera defenderse la bahía y evitar cualquier intento de desembarco en la playa de Bavel.

\section{La fortificación temporal de Bernet y Herrera}

Tras el inicio del baluarte de San Carlos como primer elemento defensivo de la fortificación permanente, tocaba acometer el segundo de los puntos a tratar con urgencia en la ciudad, su defensa provisional. Por carta del 10 de noviembre de 1691 conocemos que el maestre de campo D. Simón Bernet y el sargento mayor Don Diego de Herrera Dávila reconocieron la ciudad y dispusieron aquello más urgente de ejecutar "lo que permite la breuedad del tiempo dentro la impossibilidad del y de medios para lo mas en la conformidad de un papel que se ha formado" "l. Don Simón Bernet presentó la propuesta al rey. Expresó el pesar de la ciudad por no poner remedio a su situación transcurridos más de tres meses desde el fatal bombardeo. Igualmente mencionaba el temor a una nueva ofensiva de la escuadra francesa y la imposibilidad de defender la ciudad por más de una hora, tal y como demostraba el reconocimiento de la ciudad por parte del sargento D. Diego de Herrera. Éste por su parte debió plasmar un proyecto defensivo compuesto de memoria y plano:

"No obstante en medio de esta indisposicion y falta de armas y municiones paso el supp ${ }^{\text {te }}$. a delinear y preuenir según le dicto la ocurrencia y sus experiencia aquella defença que al presente la podra hazer mas fuerte, cuyo dictamen admitieron y recibieron gustosos el Gouernador Nobles y moradores de la Plaça, que para mas exprecion presenta una copia legalizada",2.

Por desgracia, sólo ha llegado a nuestros días la relación de Bernet y Herrera. Gracias a ella podemos averiguar las intenciones del proyecto para impedir el desembarco en la playa de Bavel. En primer lugar, la construcción medieval en piedra de los lienzos de muralla seguía mostrándose perjudicial por su altura y el efecto de la artillería sobre ella. Como punto de partida se redujo su altura y se reubicó la poca artillería en buen estado que quedaba en la plaza, diecinueve piezas:

"Hauiendo reconocido la Muralla principal de la ciudad y Baluartes de ella, que son cubos, o torreones, hallaron que conuenia, se deshiciesse parte de los parapetos dellos hasta que queden a barba de la Artilleria, y assi mesmo todos los pilares, porque siendo como son de piedra, se considera ser mayor el daño, que el prouecho, que de ellos pues de resultar. Y hauiendo hallado en todos ellos, diez y nueue cañones de bronze de seruicio y de diferentes calibres, en defeto de ser corta cantidad, para fortificar todos los puestos necesarios, se deuen estos colocar en los que se siguen.

En el torreon de San Seuastian dos cañones. En el del esperon quatro, y uno de alcanse en el de nra. $S^{r a}$. de Monserrate quatro cañones en la punta del Muelle una bateria con su parapeto y dos cañones de alcanse. $Y$ en los simentos del nuevo baluarte que se quiere levantar, dos culebrinas, y a la parte de a dentro de la puerta el Muelle, dos cañones, incluyendo uno de los desgraciados que podra seruir en este puesto" 3 .

Tras distribuir la artillería y asegurar el frente marítimo fortificado, se ordenaba la rehabilitación del lienzo del mar junto al torreón de San Bartolomé. La camisa de la muralla debía estar muy deteriorada y dispuso que se volviese a fortificar. El empleo de tierra había demostrado ser más eficaz a la hora de absorber la energía cinética de los proyectiles. No provocaba el efecto de metralla de los materiales pétreos, más perjudicial que beneficioso. La tierra del interior de la muralla, protegida por una camisa de piedra, se desplomada al ser abatida y generaba un talud natural que permitía el acceso por la brecha. El remedio era sencillo pero llegaba con muchos años de retraso a las murallas alicantinas. Dentro del relleno de tierra se generaba un entramado de madera resinosa que armaba la fábrica.

El nuevo lienzo que unía el torreón de San Bartolomé con el nuevo de San Carlos se ejecutaría de ocho palmos. El baluarte de San Carlos se estaba ejecutando y en la relación de obras de noviembre de 1691 se advertían 
algunas indicaciones de su ejecución, tal como el empleo de la estacada para impedir el desembarco en la plaza de las barcas:

"Para poner en defensa el Burgo de San francisco, que es el amenazado, por ser inmediato al desembarco, se necezita de que en la plaza de las horcas, se descubra el paredon que ay en la orilla del mar, desde el Torreon de San Bartolome, hasta la Casa de Ybarra, y se fortifique para impozibilitar la entrada.

Desde el Messon de la Balceta, hasta el baluarte nuevo, por la Orilla del mar, se ha de hazerr una estacada con su parapeto de ocho palmos de alto, y en llegando a la punta del baluarte, se ha de hazer un diente a la estacada para entrar en el, siguiendo la estacada hasta el angulo de la espalda del Baluarte, y en sus caras, y en un flanco se leuantara su parapeto, y se le ha de poner la estacada, porque en el estado pressente le facilita el desembarco al enemigo, por no estar en su perfeccion, y los medios y cortedad del tiempo, no da lugar a ello y la bateria de los dos cañones, arriua mencionados, se pondran en lo mas auanzado al mar con su esplanada de tablachos".

Para una mejor defensa el emplazamiento del cerco enemigo debía alejarse lo máximo posible. Para ello, se recurría a ensanchar fosos y colocar obstáculos defensivos convertidos en objetivos prioritarios, y por tanto, retrasando la toma de la plaza. Bernet y Herrera describieron un foso de veintiocho pies de ancho con plaza delante, con origen en el ángulo de la espalda del baluarte de San Carlos. En medio del terraplén que se unía al segundo baluarte, nombrado "calle del Pal" actual calle de Rafael Terol- proponían cerrar el acceso a la ciudad con un puente, empleando tres cañones para la defensa de la puerta:

"Y desde el angulo de la espalda del referido Baluarte, hasta el huerto de Dionis Blasco, se ha de abrir un fozo de veinte y ocho piez de ancho, y doze de profundo, con plasa alante del fozo, $y$ en la mediania del que corresponde a la calle del Pal se hara una puente capas de passar carros, con una barrera con su estacada y un torno para la gente de apie, quedando seruido con esto el Burgo de san Fran ${ }^{c o}$. y junto a la puerta en el terreno superior, tres cañones de hierro.

Arriua en dicho huerto se colocaran seis cañones de hierro por permitirlo el terreno, para limpiar parte de la marina, cargandolas con balas de Mosquete, y en una cassilla que ay en el mismo huerto se pondran las municiones necesarias para esta bateria, y otra que se ha de poner en el terrapleno del huerto de Berardo con otros dos cañones de yerro y andando desde la cassita de las municiones por dentro del Huerto, hasta el camino de elche seguarnecerá de Mosqueteros",5.

Por último, en el camino de Elche, "desde la cassa de Dionis, hasta la parte del huerto de Berardo" se ejecutaría una barrera con torno para salir la gente a pie. También era necesario condenar algunos pasos y puertas para controlar los accesos a la parte trasera del arrabal de San Francisco y a las construcciones en paralelo a la muralla del Vall:

"La Puerta de la cochera de Berardo, en el huerto se ha de condenar y en este Huerto se ha de hazer una banqueta, para poder disparar la gente que la guarnecera por la pared de las parras.

Se ha de condenar el callejon que esta desde la Torre de $S^{n}$. fran ${ }^{c o}$. a la puerta del huerto de Berardo, que esta en la plaza de este conuento y en el, y demas puestos, se han de abrir troneras hasta el Barranquet.

Esto es quanto a la defensa del Burgo, por si el enemigo lograsse el desembarco "6.

Sorprende que las medidas expuestas servían realmente de muy poca defensa, probablemente para retener un avance enemigo durante un par de días una vez desembarcado. Como hemos podido leer, la fortificación temporal se reducía a movimientos de tierra adelantados a la nueva circunvalación. De hecho el foso de la fortificación temporal se ejecutaba por donde debía discurrir la base de la fortificación permanente, por lo que su ejecución no se hacía en balde:

“(...) $m a^{s}$ mente quando su execucion ni existenzia no embarazaran que se pueda 
fabricar asta su ultima perfeccion la otra fortificaz ${ }^{o n}$. permanente por que haviendo de dorrer entrambas por unas mesmas líneas el fosso de la temporal puede servir de zanja para los fundamentos de la permanente y con el aprovecham $^{\text {to }}$. de la tierra y materiales que a hora se hande preparar se ahorrara gasto considerable para la otra quedando formados los terraplenos y parapetos en parte de su espesor porporcionado con capacidad de defnderse de qualquier acometimiento improvisto q es el que oy puede rezelarse no siendo factible que por mar venga $\mathrm{Ex}^{\text {to }}$. con aparatos para sitio formal aproches Vaterias y ataques y parece que quando el fervor con que aquel Pueblo y otros circunvez $z^{o s}$. Han formado a su cargo la fortificaz ${ }^{\text {on }}$. Confio que por todo Mayo este muy adelantada y que si el enemigo se mueve antes de estar toda la superficie en defensa podrá con estacadas y cestones suplirse lo que faltare y su coste podrá sacarse del Jornal de Villa con algo mas que se le añada" "8.

Al margen de la fortificación temporal que serviría para un posterior empleo en la fortificación permanente, Bernet y Herrera proponían atronerar los tres frentes de la casa del Bavel y hacerles un parapeto sobre el terrado, capaz de defender a cien hombres (los que estimaron necesarios para defensa y guarda de esta parte de la ciudad). A ellos sumaban doscientos caballos veteranos y una trinchera guarnecida de cuatrocientos hombres:

" $Y$ con una trinchera, desde el Huerto de $D^{n}$. Feliciano Pasqual, hasta el huerto de Just, que esta mas alla de los Banets, que el terreno nos es favorable, guarnecida de quatrocientos hombre, se impidira, el que no conciga el enemigo el dessembarco, y para guardar el Burgo de $S^{n}$. Francisco, y guarnecer las referidas fortificaciones, se necesita de mil y ducientos hombres sin los retenes (...) ","

El rey ordenó el 26 de noviembre de 1691 que en la ciudad de Alicante se tuvieran diferentes conferencias a cargo de oficiales y personas con práctica en materia de fortificación. Pasarían cuatro meses hasta que el marqués de Castel Rodrigo solicitara el 4 de marzo de 1692 que se llevaran a cabo y que interviniese el gobernador y D. Pedro Joseph Borrull, oidor y consejero de Castilla (Giménez, 2007) ${ }^{10}$. En éstas se propusieron las disposiciones que con más urgencia debían realizarse en la ciudad para mejorar su fortificación y las resumieron en cuatro puntos:

“(...) y los papeles que me han remitido sobre esta dependencia, resulta que se reduzen âquatro puntos, el primero es de la fortificazion $y$ defensa que necesita de subsistencia permanente con la fabrica de un reçinto fuerte que cubra toda la çircunferençia de la Ciudad y sus Arrauales.

El segundo âque se preuenga una fortificaz ${ }^{o n}$. temporal que pueda defenderla en caso de que la Arma ${ }^{\text {da }}$. Enemiga infestare estas costas y quisiere intntar âlgun desembarco.

el tercero â la fabrica de las cassas del Ayuntam $^{\text {to }}$. De la Ciu ${ }^{d}$. y carzeles publicas que han quedado ârruynadas del todo con preçision de hauerse de fabricar de nuevo desde la primera piedra y compranse las alaxas que fueren inesxusables $p^{r a}$ la decençia y adorno de aquel Magistrado.

$Y$ el quarto a la introduccion de Armas Muniçiones y bastimentos (...)",ll.

El virrey explicaba claramente cómo se recaudarían los fondos para llevar a cabo la fortificación permanente de la ciudad. El plan económico suponía gran austeridad, llegando a paralizar las obras del nuevo hospital. De las rentas de la ciudad se obtenían dos mil libras, de los ocho dineros que se otorgaban a obras de las iglesias. Dos se aplicarían a la fortificación. De igual forma se recaudaría de las sisas de la carne, del comercio del esparto, de los abastos del tocino y los cabritos, salinas de la Mata, bullas de la Santa Cruzada, impuestos sobre mercaderías extranjeras, derecho del tiraje de levante, etc.

La situación era desastrosa. La falta de medios con que se hallaban los reales cofres iba en aumento y no era de esperar que la situación mejorara "en las angustias de este tiempo también es imposible que se adelante la nueva fortificazion que llevo referida"12. Por esa razón se juzgaba muy importante y precisa la 
fortificación temporal delineada por los sargentos mayores D. Simón Bernet y D. Diego de Herrera, cuya traza no se conserva.

Según el gobernador y D. Pedro Joseph Borrull, el coste de las obras a ejecutar ascendía a 150.000 libras $^{13}$, de las cuales 50.000 se destinaban a la "restauración y nueva edificaz" de las cárceles públicas y casas del ayuntamien ${ }^{\text {to }}$. de la ciu c $^{\text {. y compra de las alaxas }}$ de que necesitase para el decente ornato de su Magistrado"13. La circunvalación se valoraba pues en 100.000 libras, cifra muy similar a los "ochenta o noventa mil ducados"14 asignados por Pedro Juan Valero y Jose Castellón.

Se trataba de mucho dinero y no estaba claro qué parte del mismo se destinaba a la fortificación temporal y cuál a la permanente. Es por ello que el Consejo de Aragón solicitó al marqués de Castel Rodrigo precisar "donde empieza, y donde âcaba la fortificaz $z^{\text {on }}$. de Alicante, y que costara el reçinto que llaman temporal" ${ }^{15}$ El 1 de abril de 1692 el virrey respondía que se hicieran los fosos, terraplenes y parapetos en tierra como en otras plazas. Pero Alicante contaba con tierra demasiado árida y salina, donde la capa vegetal que protegía las obras en tierra no podía crecer:

"A lo primero no tengo q responder hauiendo embiado la planta, puesto que en ella se veen siete valuartes enteros, $y$ dos medios q empiezan al mar y âcaban en la montaña deuajo del Castillo, y cerrando de oriente a poniente como alli se vee.

La fortificaz ${ }^{\text {on }}$ temporal ha de ser formada de tierra los fosos, estrada cubierta terraplen $y$ parapeto, y esto bien echo es defensible $y$ permanente como se ve en mortara y otras partes donde se formaron las plazas de zespedes y después no se han cubierto de ladrillo o piedra asta q con el tiempo se ha ido executando como el $s^{o r}$. Duque de Osuna hizo con algunos cuerpos (...)

Estas providenzias en Alicante las resiste el terreno y las imposivilita la gente. El terreno por q es todo un salinar esteril sin dedo de yerva ni forma de tener tierra travada con q se recurre a las tapias de pison, y estas acabadas dehazer se desploman como Vs veéra por la inclusa copia de la q tuve del Gov ${ }^{\text {or }}$ y luego acudió al reparo como convenia y no desacreditar la hobra ni desarruinar al pueblo.

Lo que esto puede costar no lo se ni lo pregunto porque son trascendentales y creeran me echo con la carga de las dificultades y que no se piensa en fortificarlos con permanenzia y si tal se soñasen dariamos al traste con todo (...)"15.

Es evidente que no quería valorarse la fortificación temporal. Podía haber dos razones. Primera, que Bernet y Herrera no las presupuestasen, algo poco habitual. O segunda, que se pretendiera utilizar la obra como excusa para recaudar la ayuda y emplear el dinero para otros menesteres. La planta referida es la planta del recinto de circunvalación de Castellón y Valero cuyo coste sí estaba valorado. La respuesta evidencia que a los innumerables problemas que ya tenía Alicante se sumaba otro nuevo, esta vez de orden constructivo: el desplome de los tapiales. La tierra del interior de las murallas se protegía por una camisa de piedra, pero al realizar la fortificación temporal únicamente con tierra apisonada y no existir entramado de madera resinosa que armase la fábrica, los desplomes aparecieron con suma facilidad:

"Señor, el miércoles antes de medio dia se desplomó un pedazo de las tapias de tierra q. dirigió $D^{n}$. Diego de Herrera y entre quatro y cinco de la tarde se desplomó otro pedazo q. entrambos tendrán de latitud ciento y cinq ${ }^{\text {ta }}$. Pies con poca diferencia con catorze de alto. Quedase reparando con toda aplicaz ${ }^{o n}$. Y espero lo estará breuem ${ }^{\text {te }, 16}$.

El dinero invertido servía de muy poco si por mínima que fuera la obra no era capaz de aguantarse a sí misma. Parece que los medios propuestos para las necesidades de reparos de las fortificaciones y guarda de la ciudad no fueron suficientes o jamás se llegaron a librar. No se habían podido concluir las fortificaciones temporales, ni tampoco se tenían las municiones y piezas de artillería necesarias:

"La Ciudad con estas experiencias, recelando otras, y considerando la suma importancia de su 
defensa, llevada del amor, zelo, y fidelidad, que siempre ha professado à V.M. y su Real servicio, ha sicurrido varios medios, q puedê côducir al logro de efectuarse, y hazerle en ella una cumplida, y permanente fortificacion, que assegure su defensa, y custodia en todos, y sus mas utiles resguardos, para el aumento, y côsuelo general de todos sus naturales, animosos de sacrificar sus haziendas (...)

Últimamente supone, que las urgencias, que precisan al reparo, y defensa de la Ciudad, se reducen à tres puntos. El primero, à lo que es de su fortificacion permanente para en adelante, $y$ de su continuada subsistêcia. El segundo, en quanto mira à la defensa prompta para resistir las próximas, y probables invasiones, que se pueden temer en las Primaveras. El tercero, en orden à las fabricas de las casas de la Ciudad, y Carceles publicas, que han quedado arruinadas del todo, con precisa urgencia de fabricarlas de nuevo desde la primer piedra (...)

Ademas de dichos reparos promptos, necessita la Ciudad, con la misma urgencia de armas, municiones, y bastimentos (...) y hasta el dia de oy no ha logrado la Ciudad obtener cosa de lo que se le concedió, lo qual, si huviera conseguido, con lo que ha procurado reparar por si (de que se hará representación) parece quedaría al prompto con algun resguardo para las invasiones temidas en las Primaveras, no permitiêdole dexar la otra fortificacion firme, $y$ permanente para la cumplida defensa (...) pareció que quedaría concluida del todo la fortificacion temporanea, y la ciudad con bastante municiones de boca para cautelar otra invasiô, mas como, ni se percibieron los quinientos doblones, ni el jornal de Villa llenò todas las esperanzas que se prometían, no queda la fortificacion temporanea todos los cabales, $n i$ menos se han podido recoger bastimentos (...) $)^{, 17}$.

Como era de esperar, el rey tomó la decisión de ejecutar la fortificación temporal en la forma propuesta. Lo que menos costaría a las arcas y lo que la ciudad ejecutaría sin dilación, por carecer de defensa efectiva ante las noticias de amenaza real de la armada francesa por el Mediterráneo. La ciudad se había manifestado en contra de los arbitrios propuestos por Valero, cuyo proyecto había sido defendido por el gobernador Jaime Antonio Borrás (Rosser, 1990). Así pues, proponía nuevos medios económicos para poner en guarda y defensa su fortificación. Al mismo tiempo, en septiembre de 1693, el nuevo gobernador de Alicante, D. Joseph de Borja (Bernabé, 2008) ${ }^{18}$, informaba sobre el estado en el que se hallaba la plaza.

\section{Conclusiones}

El ataque francés sufrido por la ciudad de Alicante en 1691 y los episodios bélicos posteriores de la Guerra de Sucesión, plasmaron la incapacidad de las fortificaciones alicantinas tanto por el frente marítimo como por el interior.

Ante la escasez de medios económicos y el bajo apoyo de la Corona para la modernización de las estructuras defensivas de Alicante, después del bombardeo francés, la ciudad de Alicante llevó a cabo dos actuaciones defensivas: las obras del Baluarte de San Carlos, construido bajo la traza de un ingeniero cualificado como Ambrosio Borçano, único elemento de fortificación permanente ejecutado; y la fortificación temporal diseñada por Bernet y Herrera. Ambas tuvieron la misión de evitar nuevos desembarcos por el frente marítimo de Bavel, tan agravado por el bombardeo francés.

El desfase entre las obras de fortificación de Alicante y los avances en las nuevas técnicas constructivas siempre fue notable. Los avances de la artillería superaron con creces los tiempos de ejecución de las obras defensivas.

\section{Notas}

(1) ACA. Consejo de Aragón. Leg. 674. Fol. 47/43 Carta de 10 de noviembre de 1691.

(2) ACA. Consejo de Aragón. Leg. 674. Fol. 47/45 Carta de 10 de noviembre de 1691.

(3) ACA. Consejo de Aragón. Leg. 674. Fol. 47/46 Carta de 8 de noviembre de 1691. Copia en AMA. Arm. 11. Lib. 7. Llibre de cartes escrites de la Ciutat de Alacant de 1691. Fol. 153 y ss. 
(4) ACA. Consejo de Aragón. Leg. 674. Fol. 47/46 Carta de 8 de noviembre de 1691. Copia en AMA. Arm. 11. Lib. 7. Llibre de cartes escrites de la Ciutat de Alacant de 1691. Fol. 153 y ss.

(5) ACA. Consejo de Aragón. Leg. 674. Fol. 47/46 Carta de 8 de noviembre de 1691. Copia en AMA. Arm. 11. Lib. 7. Llibre de cartes escrites de la Ciutat de Alacant de 1691. Fol. 153 y ss.

(6) ACA. Consejo de Aragón. Leg. 674. Fol. 47/46 Carta de 8 de noviembre de 1691. Copia en AMA. Arm. 11. Lib. 7. Llibre de cartes escrites de la Ciutat de Alacant de 1691. Fol. 153 y ss.

(7) El Jornal de la Villa para la fortificación temporal consistía en pagar tres ducados por cada vecino, exceptuando los eclesiásticos y los pobres.

(8) ACA. Consejo de Aragón. Leg. 556. Fol. $12 / 52$.

(9) ACA. Consejo de Aragón. Leg. 674. Fol. 47/46 Carta de 8 de noviembre de 1691. Copia en AMA. Arm. 11. Lib. 7. Llibre de cartes escrites de la Ciutat de Alacant de 1691. Fol. 153 y ss.

(10) Pedro José Borrull fue catedrático de Código e Instituía de la Universidad de Valencia entre 1669 y 1685 antes de ocupar la asesoría del justicia criminal de Valencia y ejercer como asesor del Portanveus del General Gobernador de la capital. El 12 de septiembre de 1689 fue elegido para oidor de causas criminales en la Audiencia foral, interviniendo en la represión de la Segunda Germanía.
(11) ACA. Consejo de Aragón. Leg. 556. Fol. 12/50-58.

(12) ACA. Consejo de Aragón. Leg. 556. Fol. $12 / 52$.

(13) ACA. Consejo de Aragón. Leg. 556. Fol. $12 / 53$

(14) ACA. Consejo de Aragón. Leg. 556. Fol. $12 / 52$.

(15) ACA. Consejo de Aragón. Leg. 556. Fol. $12 / 59$.

(16) ACA. Consejo de Aragón. Leg. 557. Fol. 36/9. Carta del 1 de abril de 1692.

(17) ACA. Consejo de Aragón. Leg. 557. Fol. 36/9. Carta del 1 de abril de 1692.

(18) ACA. Consejo de Aragón. Leg. 557. Fol. 36/10. Carta del 9 de marzo de 1692 .

(19) ACA. Consejo de Aragón. Leg. 557. Fol. $36 / 14$.

(20) D. Josep de Borja Llansol, conjuntamente con la titularidad de la Gobernación, fue nombrado "Administrador de los derechos Reales de V.Mgd. en dicha ciudad de Alicante y de las Salinas de la Mata y Orihuela, con el sueldo de trescientos escudos al mes". Ostentará el cargo desde 1693 hasta 1700, en Bernabé Gil, D. (2008). "Nobles valencianos en el servicio regio. La provisión del oficio de portantveus de gobernador ultra sexonam en la edad moderna. Revista de Historia Moderna", en Anales de la Universidad de Alicante $\mathrm{N}^{\mathrm{0}} 26$, pp. 13-60.

\section{Referencias}

Bernabé Gil, D. (2008). "Nobles valencianos en el servicio regio. La provisión del oficio de portantveus de gobernador ultra sexonam en la edad moderna. Revista de Historia Moderna", en Anales de la Universidad de Alicante $\mathrm{N}^{\circ} 26$, pp. 13-60.

Giménez López, E (2007). "El exilio de los borbónicos valencianos”, en Revista de Historia Moderna: Anales de la Universidad de Alicante, $\mathrm{N}^{\circ}$ 25, pp. 11-51.

González Avilés, A.B (2014). "El proyecto de fortificación de Castellón y Valero de 1688 para la plaza de alicante", in Biblio $3 W$, Vol. XIX. 
Pérez Millán, M.I; González Avilés, A.B.; Echarri Iribarren, V (2014). "The fortification of Alicante designed by the engineer Ambrosio Borçano", in Wit Transactions on the Built Environment, vol. 143.

Rosser Limiñana, P (1990). Origen y Evolución de las Murallas de Alicante. Ayuntamiento de Alicante. Conselleria de Cultura, Educació i Ciencia. Generalitat Valenciana. Alicante. p. 115. 